\title{
Les voitures dans l'œuvre de Pascale Kramer : entre vents de liberté et rouages tragiques
}

\author{
Marion RosseleT \\ Critique littéraire
}

\begin{abstract}
L’omniprésence des voitures frappe dans l'œuvre de Pascale Kramer. On n'y marche pas ; on y roule constamment. Les coups de klaxon fusent, les personnages s'observent dans le rétroviseur, des sorties d'autoroute sont manquées, des accélérations soudaines provoquent des frissons. Au cœur de situations familiales étouffantes, les véhicules jouent un rôle pluriel que l'article se propose d'explorer. A partir de quatre textes Les Vivants (2000), L'Implacable Brutalité du réveil (2009), Un homme ébranlé (20II) et Gloria (20I3), il montre comment l'automobile se révèle à la fois un opérateur puissant de la tragédie, en catalysant les forces psychologiques et sociales mises en branle, et un moyen d'échapper à ces forces, de remettre la vie en mouvement, de s'étourdir, de fuir l'enserrement.
\end{abstract}

Keywords : Pascale Kramer, voiture, force, mouvement, tragique, fuite

Ça roule dans l'œuvre de Pascale Kramer. Les voitures, aux côtés de quelques autres véhicules, y sont omniprésentes. Avec les logements, elles sont un lieu essentiel de l'univers dans lequel évoluent les personnages. Elles font office de trait d'union entre l'intérieur des maisons et la ville ou la campagne désolée, entre le chez-soi et le chez-autrui, mais elles constituent aussi un espace en tant que tel, en mouvement, avec pare-brise, rétroviseurs, sièges avant et sièges arrière. Elles permettent d'aller d'un point à un autre, de virer de bord, de fuir, de se retrouver dans la solitude ou dans un mode relationnel spécifique de par la disposition des corps dans l'habitacle et la présence du paysage qui défile. Elles sont aussi le marqueur social d'une classe moyenne inférieure française - celle, peut-être, que l'on a retrouvée en 2018 dans les rues habillée de gilets jaunes suite aux taxes introduites sur le prix du carburant, sans mesures sociales de compensation, par le gouvernement Macron.

Étant donné que les personnages communiquent peu et ne marchent que très rarement, l'automobile est souvent l'unique mouvement possible dans une situation étriquée. De fait, les scènes de voitures jouent un rôle majeur dans les dispositifs narratifs mis en place par Pascale Kramer. Ambivalentes, elles font avancer l'histoire en provoquant des ouvertures, mais surgissent aussi comme des opérateurs de la mécanique qui broie les personnages. Les voitures sont présentes dans nombre de romans, selon une déclinaison de modalités que cet article se propose d'explorer. Le texte Les Vivants (2000) a été le déclencheur de ma réflexion sur le sujet. Sans prétention 
à l'exhaustivité tant le motif est récurrent dans l'œuvre de Pascale Kramer, trois romans plus récents seront convoqués, à savoir L'Implacable Brutalité du réveil (2009), Un homme ébranlé (201I) et Gloria (2013).

Les Vivants débute sur une scène automobile : à la fenêtre de la maison familiale isolée au bord d'une route, Benoît guette l'arrivée en voiture de sa grande sœur Louise, du mari Vincent et de leurs deux enfants. L'adolescent, qui vit encore avec sa mère, dissèque les arrivants du regard, admire la beauté et la patience de sa sœur, constate l'énervement de Vincent. Étant donnée la configuration en surplomb, il peut s'autoriser à observer sa sœur sans restriction. Ce regard perdurera tout au long du livre, Louise étant souvent appréhendée par Benoît comme une image ou une émettrice de sons ${ }^{1}$, sans questionnement sur son intériorité qui demeure le point noir de l'histoire. En premier lieu, la voiture est l'occasion d'observer l'autre à son insu lorsqu'il arrive chez soi. A l'intérieur du véhicule, cette fonction réapparaît par la suite via les rétroviseurs qui permettent à Benoît de guetter les expressions de sa sœur, assise à l'arrière.

Après cette arrivée, l'ambiance familiale se fige et se fait lourde. Vincent est colérique, Louise d'une abnégation sans relief. Au cœur de l'immobilité, la seule initiative de Louise et de Benoît va s'avérer fatale. Avec les enfants, ils partent tous deux prendre le soleil dans une carrière désaffectée. Là-bas, pour vaincre l'inaction dont fait preuve Louise qui sommeille en maillot de bain sur son linge, Benoît fait monter les enfants dans un vieux téléphérique, dont le mécanisme - en une « accélération considérable » (27) - lâche soudainement : les deux enfants sont tués sur le coup. Une réplique de la scène se rejouera plus tard lorsque Vincent initie Benoît à la conduite. Ce dernier accélère violemment, provoquant une sortie de route et un accident heureusement sans gravité. L'adolescent perd le contrôle des machines qui matérialisent les forces inconscientes qui le traversent.

Après le drame, Louise a « une impalpable inquiétude à quitter la maison »(I76). À aucun moment, elle ne manifeste de la colère. Anéantie par le deuil et abrutie par les calmants, elle est diaphane et comate sans cesse. C'est l'été, saison de l'absence de travail et des corps assommés par le soleil. L'air ne circule pas. Benoît n'ose plus prendre aucune initiative. La paralysie - que l'écriture rend palpable - devient insoutenable. La voiture, non sans ambivalence, crée alors les premiers appels d'air.

I La sonorité de Louise est très présente, par exemple : «Louise à ses côtés émit un drôle de claquement de langue en cherchant de l'air avec sa bouche desséchée par la chaleur et le sommeil, puis poussa un soupir qui parut l'aplatir encore davantage sur la dalle » (Kramer 2000 : 155); « Elle émit un drôle de bruit de gorge, un bruit de salive étranglée, posa ses mains bien à plat sur ses genoux et resta à regarder dans le vague, ses lèvres nacrées scellées en une moue impassible» (I78). 
De timides soulagements viennent d'abord des allées et venues de Louise, que les parents de Vincent et la mère se renvoient comme une balle de ping-pong, cherchant successivement à apaiser la solitude de leur deuil par sa présence, puis à se décharger de cette même présence devenue trop douloureuse. Jamais Louise ne conduit les engins qui la trimballent. Les maigres espoirs, dont l'automobile est le vecteur, proviennent de l'attente du changement. Au départ de Louise, « Benoît osait à peine s'avouer qu'il était soulagé de voir son désespoir s'éloigner un peu » (48). La voiture disparaît de sa vue, mais rien n'y fait, il a « l'impression d'un vide affolant » (50). Seule la perspective ouverte d'un changement, qui déçoit continuellement, allège le fardeau et permet de continuer à vivre.

L'automobile prend une fonction plus franchement salutaire lorsque Vincent propose à Benoît de faire un tour en voiture et qu'ils font grimper le compteur. Une camaraderie naît entre eux, animés du même « besoin de s'étourdir »(6I). «Dans une palpitation de musique et de vitesse » (63), Benoît ressent « un peu d'excitation » (62). Il y aura plusieurs virées, avec Louise aussi, que la vitesse rend " presque gaie » (I20). Son statut de passagère lui fait éprouver une circulation vitale tout en restant passive.

Chez Vincent, la conduite provoque peu à peu la « tentation de s'enfuir pour de bon » (62). Après un trajet, il téléphone et « sa voix tremblait encore de cette exaltation de vitesse et de liberté qui lui avait réchauffé le corps, cette sensation de vie »(I3I). Benoît, qui ne roule qu'à vélo, est un peu jaloux de l'engin dont dispose son beau-frère. Il se met alors à rénover un vieux side-car à deux places, laissé à l'abandon dans le garage. En arrière-fond s'ajoutent également une caravane, parquée non loin de la maison, et la fille qui l'occupe. Vincent et Benoît sont tous deux happés par elles, si extérieures à leur drame.

La voiture joue encore dans Les Vivants un rôle d'indice : en apercevant un véhicule, les personnages comprennent la situation avant même qu'il y ait contact avec les autres, avant même que des mots soient prononcés. Ainsi, à l'arrivée de l'ambulance devant la maison, la grand-mère prend conscience que les enfants sont morts. Elle accuse le choc seule. Ou, pour Benoît, la présence de l'homme que fréquente sa mère n'est matérialisée que par les bruits de sa voiture. Il n'ose même pas regarder le véhicule, mais entend des claquements de portière, entrevoit la lueur des phares et élabore des hypothèses à partir de ces signes².

2 Cette fonction d'indice est également prépondérante dans le roman Retour d'Uruguay (2003) où le mystère de la famille observée par le personnage principal prend toute son acuité lors des arrivées et départs en voiture de ses membres : les scènes donnent à la fois des informations conséquentes sur cette famille, mais révèlent aussi l'ampleur du mystère. Ce qu'il se passe à l'intérieur du véhicule demeure hors de la portée du personnage principal. 
Au fil du texte, une logique implacable se met en branle qui conduit à la fuite finale de Vincent. Kramer fait pressentir et même souhaiter cette issue aux lecteurs et lectrices. Malgré sa dureté, on finit par comprendre le réflexe de survie qui consiste à fuir Louise. Le regard sur Vincent change : on ressent intimement la nécessité qu'il y a à rester en vie et à suivre la seule voie qui insuffle de l'énergie. Comme le préfiguraient le side-car à deux places et les scènes de voiture qui agissent à la fois comme des condensés et des catalyseurs, Vincent part en décidant d'emmener Benoît et de laisser Louise derrière eux. Le livre se termine sur ce départ en voiture : Benoît, aux côtés de Vincent, se trouve cette fois embarqué à l'intérieur du véhicule, tandis que restent à la maison la mère aux côtés de Louise, dont on doute qu'elle puisse survivre à ce départ, tout en espérant qu'il s'avère - au contraire - salvateur.

Dans L'Implacable Brutalité du réveil, la voiture d'Alissa, jeune mère dévastée par la naissance de sa fille, la responsabilité et la solitude qu'elle provoque, joue également un rôle symbolique important. Il y a le siège arrière, sur lequel Alissa manque plusieurs fois d'oublier son bébé ou auquel elle ne l'attache pas. Il y a la sortie d'autoroute volontairement manquée. Dans le rétroviseur, c'est elle-même que la jeune mère regarde. Elle mesure combien le retour en arrière est impossible. La voiture constitue néanmoins un moment d'abandon et de fuite de ses responsabilités. Dans l'habitacle, protégée par la carrosserie, elle ne répond pas aux téléphones de ses proches qui l'assaillent. Elle fait la sourde oreille, se révolte, retrouve une part de liberté. L'automobile est pratiquement son unique extérieur, la seule alternative au tête-à-tête à domicile avec son bébé. Elle s'engouffre « comme on lâche prise dans le flot de voitures qui filait par la brèche creusée dans la montagne » (Kramer 2009 : 4I) et se laisse aller jusqu'où la mènent ses pulsions.

Lorsque Claude, le personnage d'Un homme ébranlé malade du cancer, décide de rencontrer un fils qu'il ne connaît pas, né d'un adultère, il propose tout de suite un tour en voiture « histoire de n'être pas obligé de se regarder, de faire passer le temps » (Kramer 2011 : I4). Le véhicule permet des rapports latéraux, tout en étant divertis par le dehors. Sauf que Claude ne laisse aucune chance à ce dehors - la banlieue - qu'il critique avec amertume. Sa femme, assise à l'avant, doit vivre avec la déchéance physique de son mari, son humeur détestable et avec « cet enfant dans le rétroviseur » (I8). Les personnages sont brutaux entre eux, se klaxonnent sans cesse - le son agressif rythme leurs interactions à la sauvette, à la sortie d'un véhicule, soit pour se passer l'enfant, soit parce que les proches, effrayés par l'état de Claude et par peur de déranger, fuient aussitôt arrivés.

Autour du couple, des motos et des voitures tournent et se font menaçantes. Ancien entraîneur, Claude suscite de l'animosité. Des gens lui en veulent suite à une bagarre survenue entre ses joueurs dans les vestiaires. 
La violence latente à chaque page du roman éclate finalement dans la rue : les jeunes des banlieues boutent le feu aux voitures. Des sirènes tonitruent dans la nuit. Claude, sa femme et l'enfant sont fascinés par ces images télévisées de véhicules en flamme. Les personnages sont enserrés dans des mécaniques psychologiques et sociales qui, sans être symétriques, se répondent, s'amplifient et s'expriment l'une l'autre. Dans ce roman, tout comme dans Autopsie d'un père (2016), il y a un conflit social qui se joue par véhicules interposés ${ }^{3}$. Seuls les cyclistes font office de distraction bienvenue et inoffensive.

Michel, le personnage principal de Gloria, possède lui aussi une voiture. Avec elle, il fait des allers-retours chez Gloria. Ces mouvements sont empreints de culpabilité, car Michel est sous le coup d'accusations diffuses. Alors qu'il travaillait dans un centre pour jeunes femmes en difficulté, il aurait fait preuve envers certaines de comportements inappropriés dont la nature demeure ambigüe. Après son licenciement, l'une de ces jeunes femmes - Gloria - reprend contact avec lui, car elle cherche à obtenir un appartement plus grand. Michel sait qu'il ne devrait pas entretenir cette relation. Au fur et à mesure qu'il s'enferre dans la situation, les scènes automobiles évoluent. L'accent est d'abord mis sur un dispositif à caractère voyeuriste : Michel observe l'extérieur depuis sa voiture en relative discrétion. Puis, on le voit retrouver son véhicule, après les visites, tout taché de poussière de tilleul et même, une fois, cambriolé. À la fin, ce sont les autres qui viennent le chercher dans son automobile, frappent sur le capot, l'attendent, furieux, adossés au véhicule. Du vecteur de son voyeurisme, la voiture se fait indice et le rend vulnérable. À l'intérieur, c'est souvent la honte de ses initiatives qui l'assaille. Il ne devrait plus se mêler de tout cela. Le véhicule est aussi le lieu d'un furtif et triste rapport sexuel, entre lui et une amie de Gloria. La configuration offre la possibilité d'un contact génital tout en évitant au maximum la relation à l'autre.

Au cœur des liens qui enferment, les voitures jouent un rôle pluriel dans l'œuvre de Pascale Kramer. Elles sont souvent le seul espoir de mouvement et de fuite, mais elles constituent également la matérialisation des forces psychologiques mises en branle, cet appareillage d'une puissance inouïe qui dépasse toujours les capacités réflexives des personnages, pourtant très développées, et qu'ils essaient de maîtriser tant bien que mal. Le trafic et les véhicules sont aussi l'expression de forces sociales, celle notamment de la jeunesse des banlieues. Dans ces tragédies contemporaines, les automobiles remplacent les chevaux d'antan, un attelage des forces inconscientes, qu'il s'agit de parvenir à monter et à guider. Le fonctionnement de l'appareil psy-

3 On retrouve aussi un tel conflit dans le Retour d'Uruguay (2003) où une bande de motards menace un père de famille et sa Volvo bleue. La tension se résout dans une course poursuite meurtrière. 
chique est au cœur de l'expérience menée par l'auteure dans l'écriture. Le tragique possède une logique propre, des rouages, des courroies de transmission, une forte inertie. Selon une conception très psychanalytique, les pulsions issues des profondeurs du psychisme opèrent et se déploient. Le trafic routier est implacable et pourtant heureusement ambivalent, voire cathartique : la musique, la vitesse et les fenêtres ouvertes sont les sources des rares espoirs et vents de liberté offerts aux personnages.

\section{Bibliographie}

Kramer, Pascale, Les Vivants, Paris, Calmann-Lévy, 2000.

-. Retour d'Uruguay, Paris, Mercure de France, 2003.

-. L'Implacable Brutalité du réveil, Paris, Mercure de France, 2009.

-. Un homme ébranlé, Paris, Zoé, $201 \mathrm{I}$.

-. Gloria, Paris, Flammarion, 2013.

—. Autopsie d'un père, Paris, Flammarion, 2016. 